\title{
Analgosedation before Less-Invasive Surfactant Administration: A Systematic Review
}

\author{
Sophie Tribolet Nadège Hennuy Diane Snyers Caroline Lefèbvre \\ Vincent Rigo
}

Neonatology Division, University Hospital of Liège, Liège, Belgium

\section{Keywords}

Surfactant administration - Less-invasive surfactant

administration · Sedation · Analgesia

\begin{abstract}
Background: Surfactant therapy is the cornerstone of respiratory distress syndrome management. "Less-invasive surfactant administration (LISA)" is now recommended for spontaneously breathing preterm infants. Analgosedation remains controversial as 52\% of European neonatologists do not use any. This systematic review aims to describe the efficacy and safety of different drugs for analgosedation during LISA. Methods: MEDLINE via Ovid, Embase, Scopus, and Cochrane Library of Trials were searched independently by 2 reviewers for studies on sedation or analgesia for LISA, without filters or limits. Results: Eight studies (1 randomized controlled trial) recruiting 945 infants were included. Infant pain was significantly reduced, with more infants evaluated as comfortable. Failure, defined as need for intubation or for a second dose of surfactant, was not different between sedated and unsedated groups. Analgosedation was associated with a higher occurrence of desaturation and need for positive pressure ventilation during procedure, but the need
\end{abstract}

for mechanical ventilation within 24 or $72 \mathrm{~h}$ of life was not significantly different. There does not seem to be any difference in clinical tolerance and complications (e.g., hypotension, mortality, air leaks, etc.). Procedural conditions were evaluated as good or excellent in $83 \%$ after sedation. Discussion and Conclusion: Analgesia or sedative drugs increase infant comfort and allow good procedural conditions, with a limited impact on the clinical evolution. Questions remain about the best choice of drugs and dosages, with the constraint to maintain spontaneous breathing and have a rapid offset. Further good quality studies are needed to provide additional evidence to supplement those limited existing data.

(c) 2022 S. Karger AG, Basel

\section{Introduction}

Respiratory distress syndrome (RDS) remains a significant problem in preterm infants. Surfactant is the cornerstone of its management and its modes of administration have been extensively studied.

In recent years, alternatives to endotracheal intubation for surfactant administration have been developed, espe- 
cially the "less-invasive surfactant administration (LISA)" method. This involves the tracheal insertion of a smalldiameter catheter to instil surfactant while the infant breathes spontaneously on nasal continuous positive airway pressure. Several studies and meta-analyses have demonstrated the effectiveness of this technique in reducing mechanical ventilation (MV), bronchopulmonary dysplasia (BPD), and mortality [1, 2]. In 2019, the European Consensus Guidelines on the Management of RDS updated its recommendations to state that "it is reasonable to recommend (LISA) as the optimal method of surfactant administration for spontaneously breathing infants who are stable on nCPAP" [3]. However, many questions remain, and current studies still explore appropriate treatment thresholds for different gestational ages (GAs), ideal catheter/device for administration, or the suitable type of surfactant [4]. The issue of analgesia and sedation during the procedure also remains controversial $[1,4-6]$.

Prior to the 2000s, tracheal intubation was usually performed on awake neonates despite several studies having revealed its association with deleterious physiological effects including bradycardia, hypertension, and intracranial hypertension [7-9]. Premedication attenuates physiological responses to intubation, shortens procedure time, and makes it easier [9]. A 2001 consensus statement for prevention and management of pain in the newborn advised for premedication in non-emergent intubation [10].

Evolutions in practices include new endotracheal tubes, use of video-laryngoscopes, and an emphasis on safe laryngoscopy [11]. Specifically in LISA, lower pressures on laryngotracheal structures from the small-bore catheter may also reduce its physiological impact.

Recent surveys revealed that at least $52 \%$ of neonatologists in Europe and 94\% in the USA do not use analgosedation for LISA $[12,13]$. Some centres have policies restricting drugs for specific indications (e.g., second attempt, more mature babies, etc.) or individualized approach $[4,14]$. Procedure performed early after vaginal birth benefits from endogenous analgesia related to high vasopressin levels [15]. A survey in Spain highlighted that all participants considered that sedative medications reduce experience of pain and discomfort, and 54\% believed that it improved procedural conditions and shortened the duration of the procedure [16]. Non-pharmacological measures for analgesia (sucrose, swaddling, environmental control, etc.) were used in all hospitals in Spain [16] and were preferred by $55 \%$ of physicians in the UK [17].

Specific conditions may explain high rates of awake laryngoscopy for LISA. An important limit is the need to maintain cardiorespiratory stability and to have a minimal impact on the respiratory drive centres. Spontaneous breathing is considered important during LISA procedure to assure the surfactant dispersion from the trachea and to allow the infant to stay on CPAP $[5,6]$. The first large RCT, the Avoidance of Mechanical Ventilation study [18], investigating LISA allowed for sedation at the discretion of the caregiver. In their online supplementary material, they briefly reported that rates of failure in infants who were actually treated with LISA were higher when premedication was used. The populations were not further described. Thereafter, most studies investigating LISA were performed without systematic analgosedation, and it was still proved to be beneficial. Adding analgosedation could therefore potentially change the beneficial outcomes. Klotz et al. [12] hypothesized that compared to invasive intubation, LISA was perceived to be less traumatic or that the maintenance of spontaneous breathing was considered paramount. The aim of this systematic review was to describe the efficacy and safety of different drug regimens for analgosedation during LISA.

\section{Methods}

Research Protocol

This systematic review was conducted in accordance with the Cochrane Handbook for Systematic Reviews of Interventions and reported following the Preferred Reporting Items for Systematic Reviews and Meta-Analyses statement for meta-analysis in health care interventions [19]. The protocol was registered in advance of data extraction with the Prospective Register of Systematic Reviews (registered on September 2020; CRD42020205365).

The initial protocol was modified on February 26, 2021, before extracting data. The focus shifted to LISA rather than both LISA and INSURE (intubation-surfactant-extubation) because of important differences between these procedures and their physiology. In addition, retrospective studies were included for a broader overview of practices.

\section{Criteria of Eligibility}

All clinical studies of LISA procedure after analgosedation were considered eligible. We included randomized controlled trials (RCTs) and prospective and retrospective cohorts published in English as well as in non-English language. In studies comparing LISA with another method of surfactant administration, the LISA arm was included if the procedure was performed after sedation. Studies on animal models, review articles, editorials, comments, and case reports were excluded.

\section{Information Sources and Search Strategy}

MEDLINE via Ovid, Embase, Scopus, and Cochrane Library of Trials were searched between inception and July 26, 2021, without any language restriction, filter, or limit. The search included $\mathrm{MeSH} /$ Emtree terms as well as free language. Search strategies are 


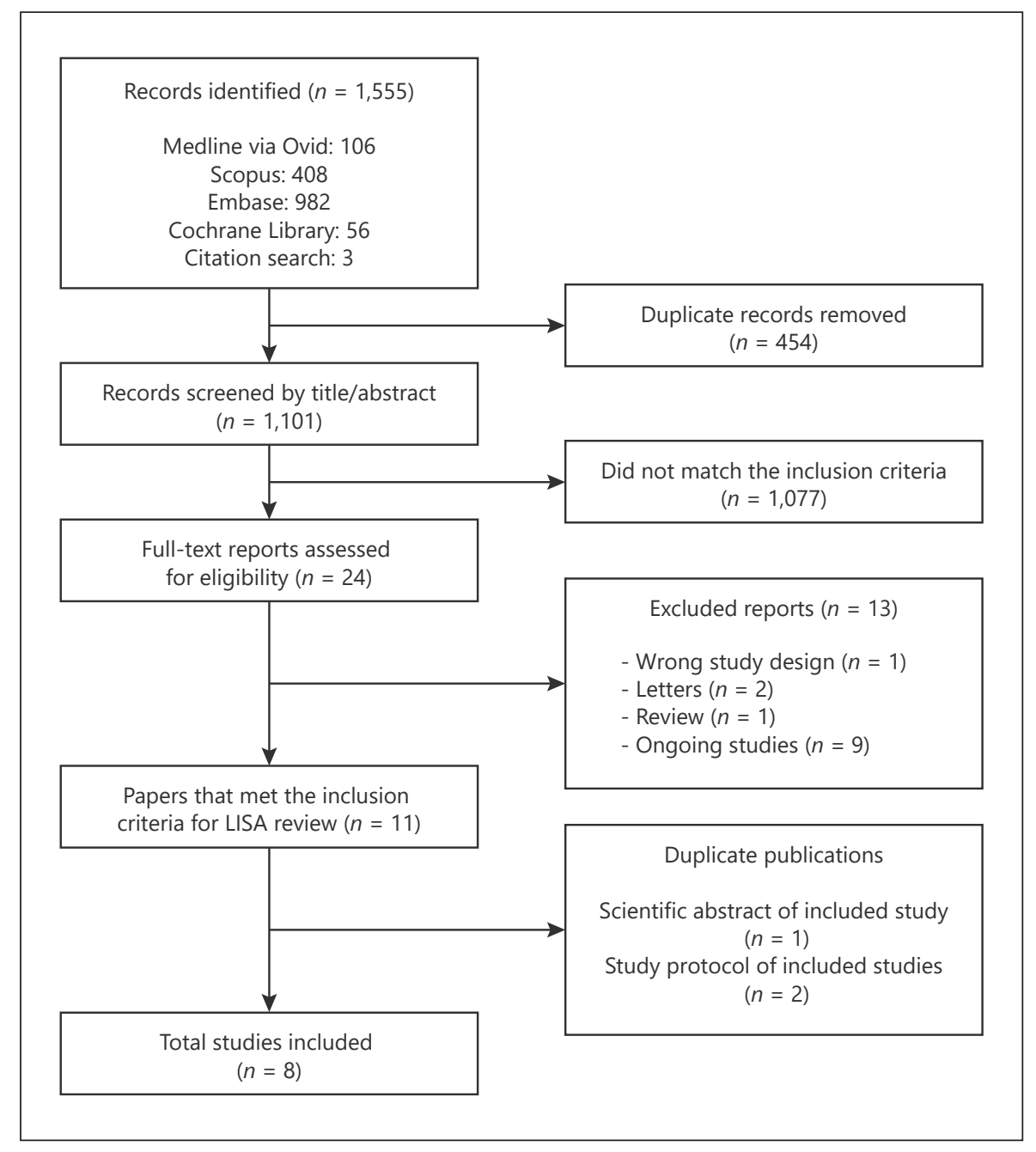

Fig. 1. Flow diagram of study selection.

available in the online supplementary material (for all online suppl. material, see www.karger.com/doi/10.1159/000521553). Subsequently, Google Scholar was searched for the grey literature. Reference lists of publications eligible for full-text review and systematic review about LISA procedure allowed for an additional "snowball search."

\section{Selection Process}

The Rayyan QCRI web app (rayyan.qcri.org) was used for a 2 -steps study selection. After exclusion of duplicates, two reviewers independently screened titles and abstracts for potentially relevant studies.

Full texts were then independently reviewed for eligibility by two reviewers. Conflicts at any step of the selection process were resolved by a third reviewer.

\section{Data Extraction and Analysis}

Relevant data were independently extracted in predetermined tables by 2 reviewers. We extracted data about the reports, the study design, the population, and the intervention. Authors were contacted for additional data, if necessary.

A Systematic Review of Analgosedation before LISA

\section{Data Items}

The selection and the importance rating of patient-oriented outcomes were determined in advance through discussion. Main outcomes were procedure failure, defined by the intubation rate and/or the need of a second dose of surfactant, and infant's comfort or pain.

Secondary outcomes focused on clinical tolerance of the procedure: occurrence of desaturation $\left(\mathrm{SpO}_{2}<85 \%\right)$, bradycardia, and hypotension (defined as a mean arterial blood pressure in $\mathrm{mm} \mathrm{Hg}$ below the number of weeks of GA). Respiratory outcomes and complications were also retrieved: air leaks, need for MV and duration thereof, oxygen therapy requirements, and duration.

Markers of procedural conditions were analysed, namely duration of procedure and the number of attempts of laryngoscopy or catheterization. Finally, surrogate markers of immediate impact on the brain, specifically cerebral oxygenation and electrical cerebral activity, were searched.

\section{Bias and Quality Assessment}

Two independent authors evaluated the risk of bias (RoB) and assessed quality in individual studies using the Revised Cochrane Risk-of-Bias for randomized trials or the Newcastle Ottawa Scales 


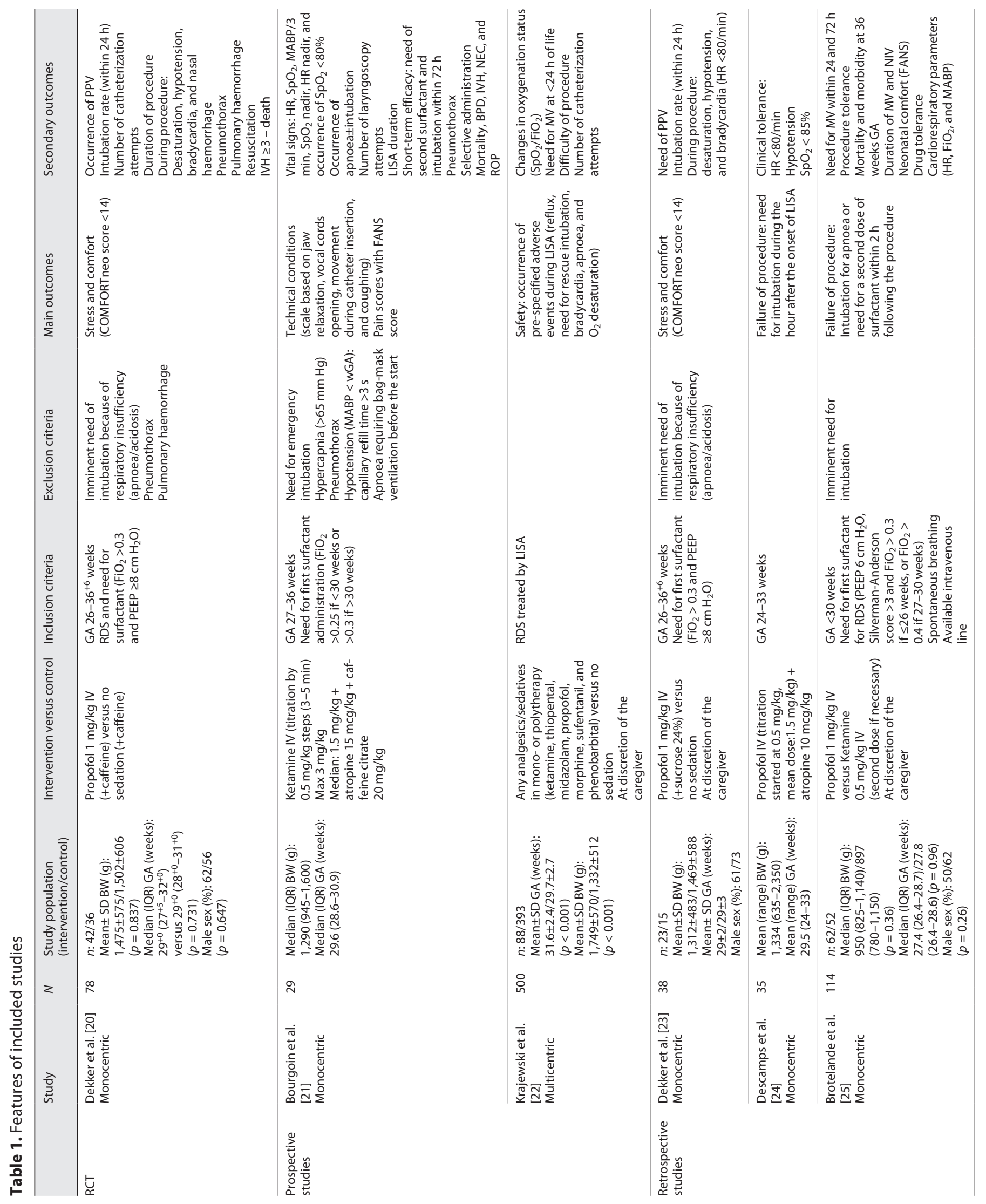




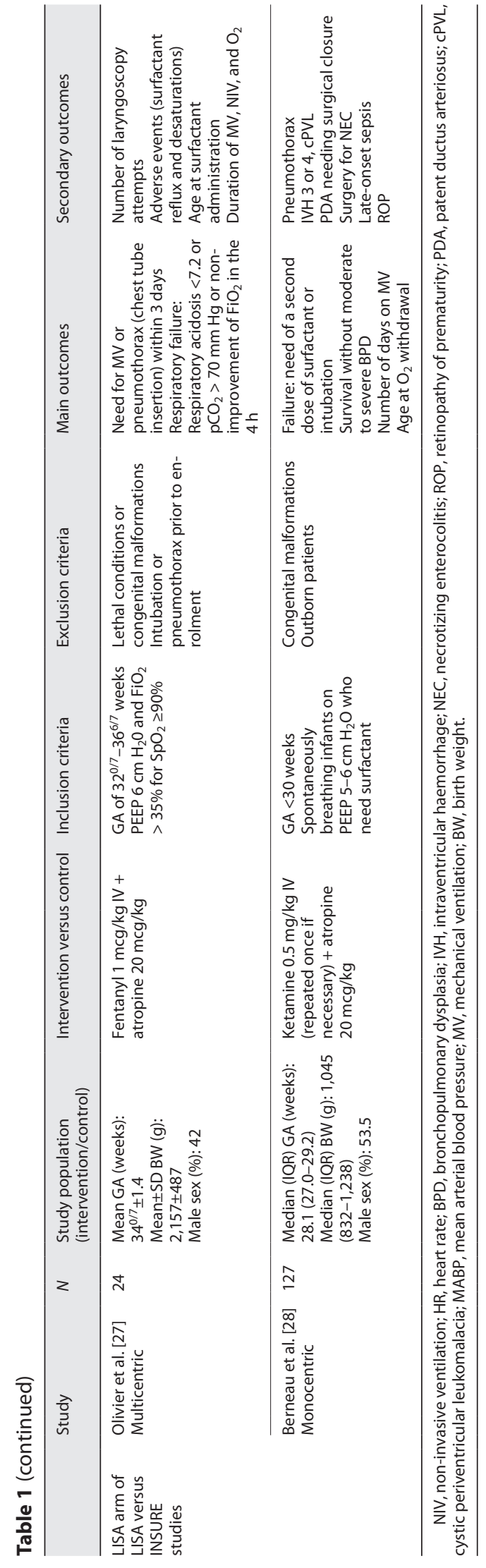

A Systematic Review of Analgosedation before LISA
(NOSs) for cohort studies. For the RCT, the following domains were assessed: randomization process, deviation from intended intervention, missing outcome data, measurement of outcome, and selection of reported results. For cohort studies, quality of selection, comparability, and outcomes were evaluated. Assessments are available in online supplementary information.

Synthesis Methods

Meta-analyses could not be undertaken given the limited number of RCTs and studies with comparative arms. We detailed data in a predefined table, according to main and secondary outcomes of each study.

\section{Results}

\section{Literature Search and Study Selection}

The search strategy produced 1,555 records. After accounting for 454 duplicates, 1,101 records were screened by the title and abstract and led to explore 24 full-text articles. Eleven references met inclusion criteria. Three were duplicate publications (one scientific abstract and 2 protocols), leaving 8 studies for analysis (see PRISMA flowchart in Fig. 1). Nine ongoing studies were also referenced.

\section{Study Characteristics}

Characteristics of the included studies are summarized in Table 1. Only one study was randomized and controlled [20]. Dekker et al. [20] studied propofol versus no sedation during LISA, with infant comfort and pain as primary outcomes.

Two prospective studies were included [21, 22]. Bourgoin et al. [21] studied the impact of ketamine on technical conditions and pain scores during LISA, without comparison. Krajewski et al. [22] conducted a nationwide cohort study and compared analgesics and sedatives such as ketamine, midazolam, propofol, sufentanil, morphine, thiopental, and phenobarbital in mono- or polytherapy to no analgosedation. Their main outcome was the safety of sedation by assessing the occurrence of pre-specified adverse events during LISA (surfactant reflux, need for rescue intubation, bradycardia, apnoea, and desaturation).

Three retrospective observational studies were included [23-25]. All used propofol as the sedative drug, in comparison once with ketamine [25] and once with no sedation [23], at the discretion of the caregiver. The third study [24] was a preliminary study without a comparative arm, prior to a multicentric RCT currently recruiting [26].

Finally, two studies comparing INSURE and LISA with fentanyl [27] or ketamine [28] premedication were 


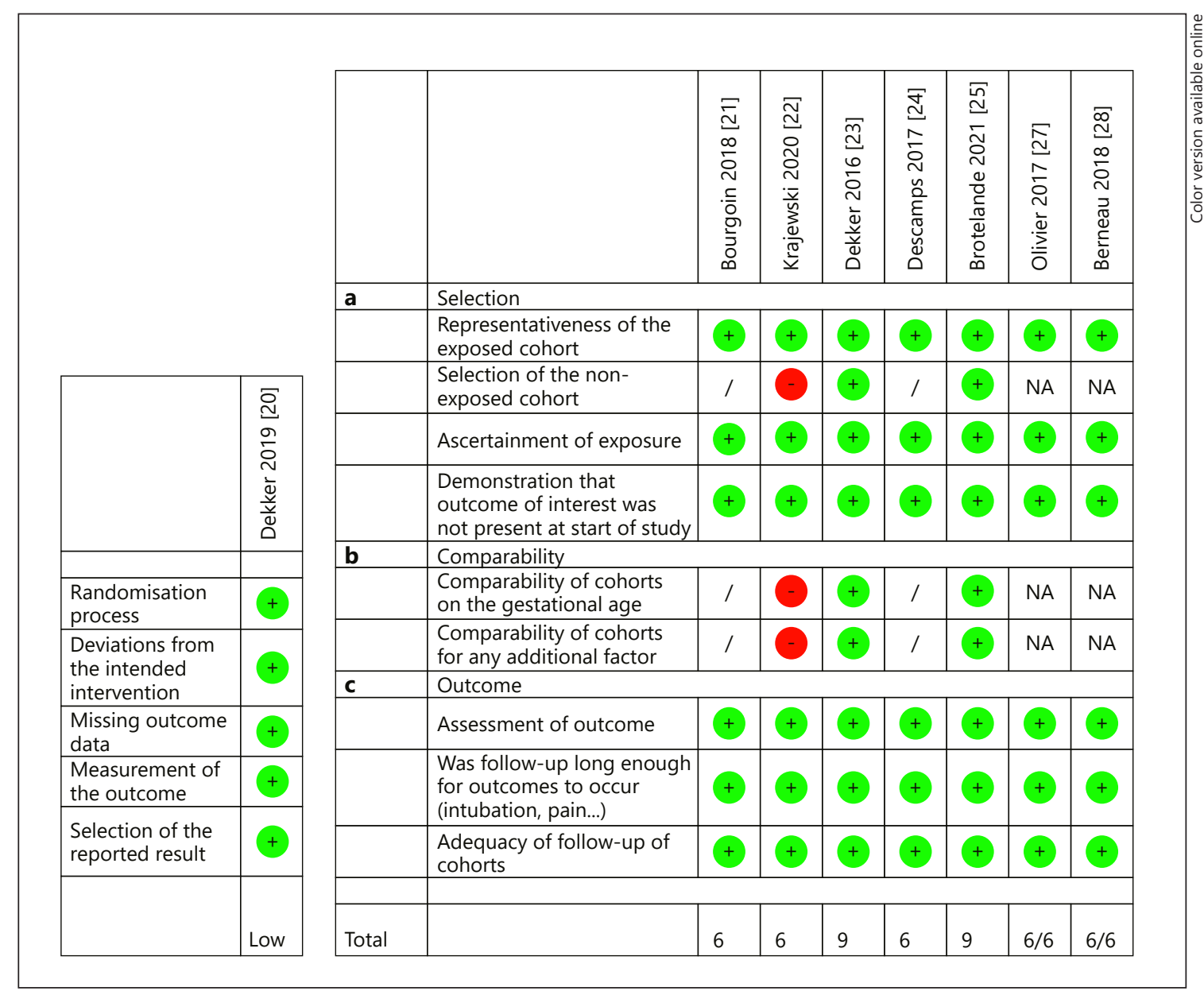

Fig. 2. RoB and quality assessment using RoB2 (Cochrane) and the NOS, respectively. RoB2, Revised Cochrane Risk-of-Bias for randomized trials.

included. The LISA arms of those trials were assessed as prospective cohorts. All studies but one [22] were monocentric.

\section{Patient Characteristics}

In total, 945 newborns were included, with study recruitments ranging from 24 to 500 infants (78 in the RCT). Three studies focused on extremely and very preterm infants $[24,25,28]$, one on moderate and late preterm [27], while the 4 others included preterm infants of all GAs.

In the RCT by Dekker et al. [20] and in cohort studies by Dekker et al. [23] and Brotelande et al. [25], groups were matched in terms of GA, birth weight, and sex. Krajewski et al. [22] reported statistically significant differences in terms of GA and birth weight $(p<0.001)$ with older and bigger infants in the analgosedation group.

\section{RoB and Quality Assessment}

The RoB of the RCT of Dekker et al. [20] was evaluated as low. Quality of the cohort studies was assessed as good, excepted Krajewski et al. [22] where differences between groups decreased their comparability. Assessments are summarized in Figure 2. There was obviously no unsedated arm in the LISA versus INSURE studies. Two other studies did not have a comparative arm either.

\section{Outcomes Analysis}

Results of the systematic review are detailed in Table 2 and summarized in Figures 3, 4.

\section{Randomized Controlled Trial}

Dekker et al. [20] found a significant reduction in the mean COMFORTneo score $(p<0.001)$ and a higher proportion of infants evaluated as comfortable during the 


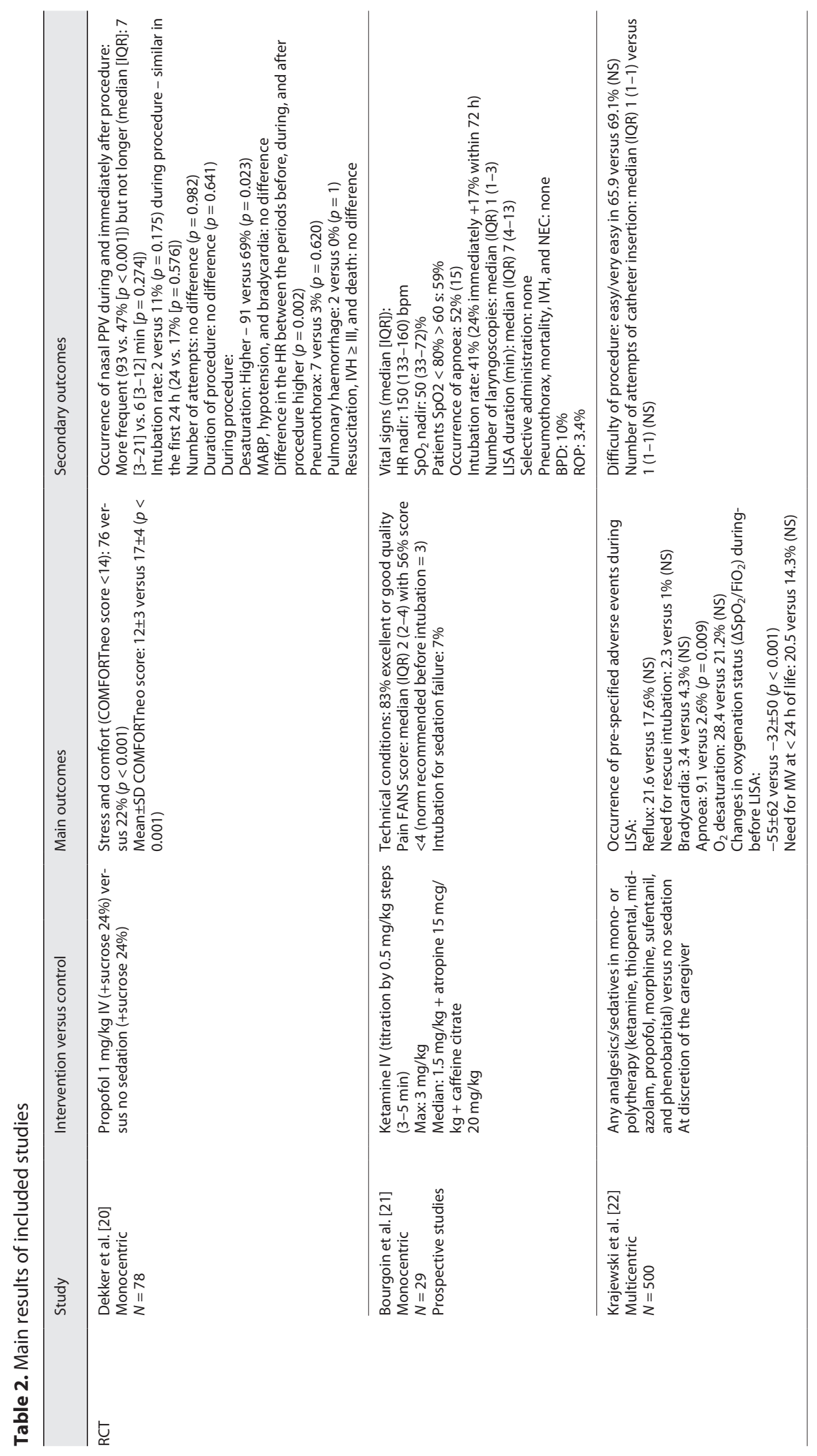




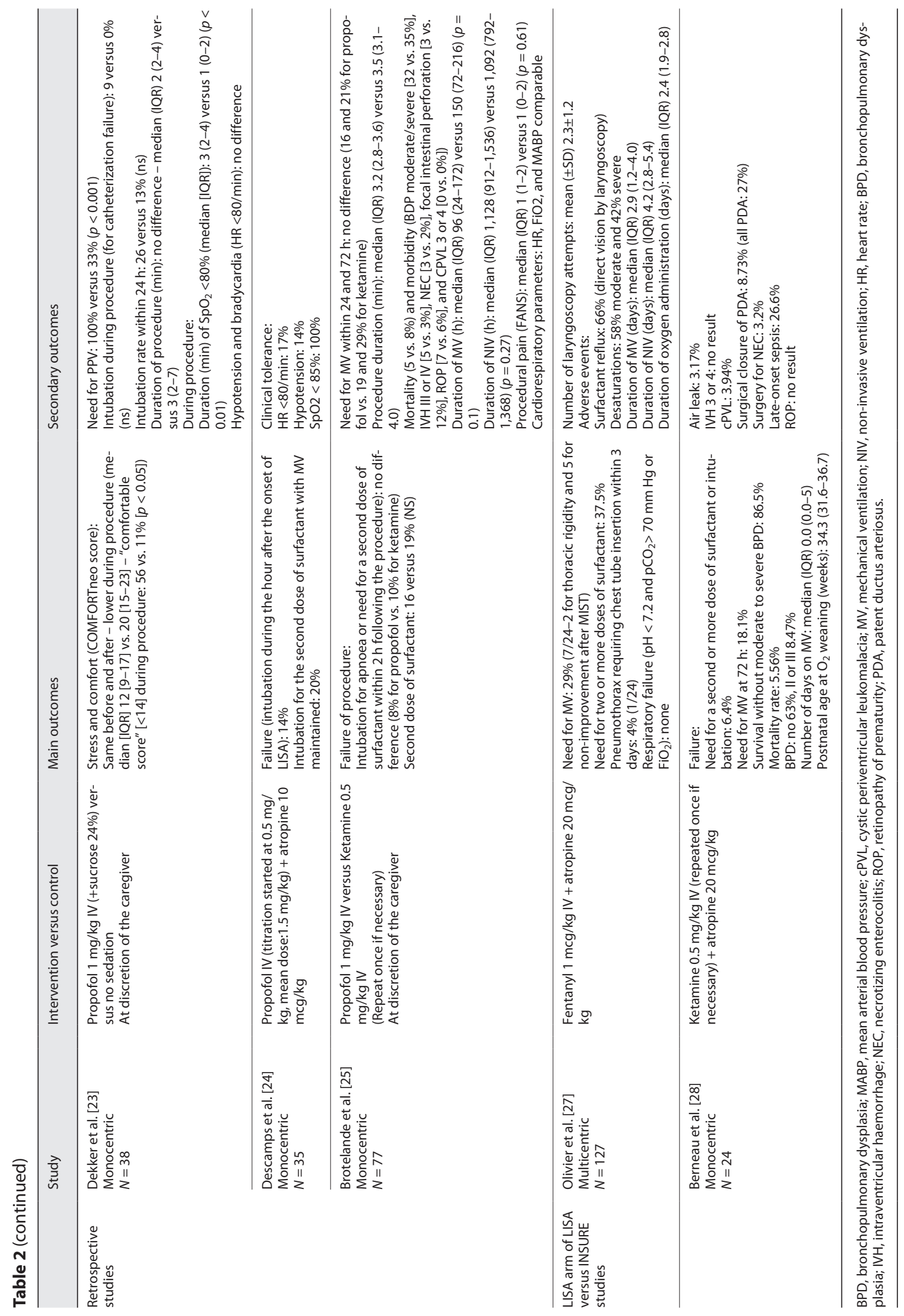




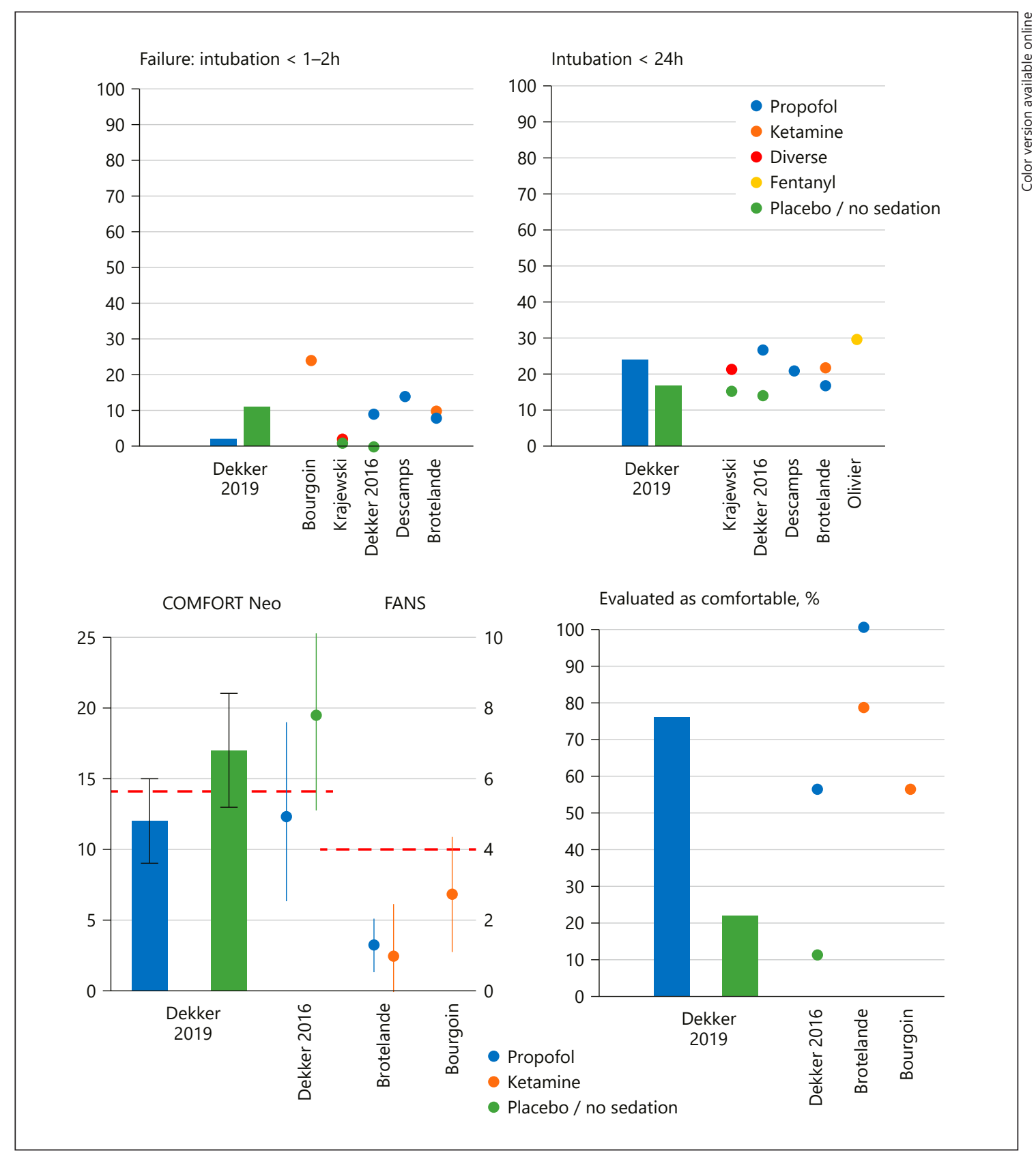

Fig. 3. Main outcomes: intubation rates and comfort and pain scores.

procedure $(p<0.001)$ after propofol compared to no analgosedation. Intubation rates were not different between groups.

Among markers of clinical tolerance, a significantly higher incidence of desaturation $(p=0.023)$ is described in the propofol group. Occurrence of hypotension and bradycardia did not differ between sedated and unsedat- ed groups. More infants in the propofol group needed non-invasive positive pressure ventilation (PPV) during procedure $(p<0.001)$. Incidence of pneumothorax, pulmonary haemorrhage, intraventricular haemorrhage grade 3 or 4 , or mortality was not different. The number of attempts for catheter insertion and the total duration of procedure were comparable. 


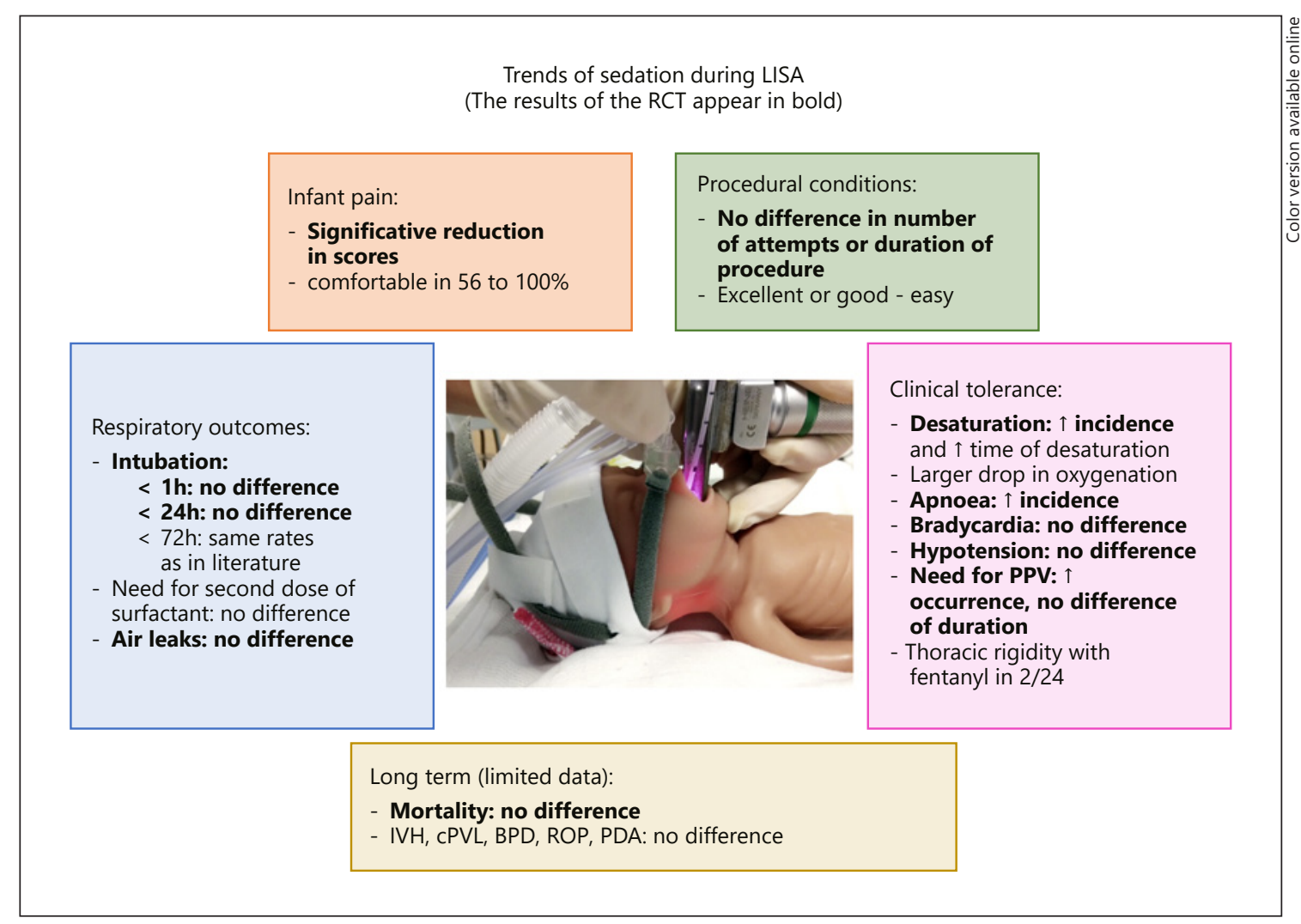

Fig. 4. Trends of sedation during LISA: a summary (the results of the RCT appear in bold).

\section{Cohort Studies}

Respiratory Outcomes. Intubation during or within 1 or $2 \mathrm{~h}$ of the procedure was often assessed as "failure" in studies. Its rates ranged from 2.3 to $24 \%$ [21-25], with no significant difference between sedated and unsedated groups [22, 23].

Need for MV within $24 \mathrm{~h}$ was not different between awake and sedated infants [22, 23]. Brotelande et al. [25] reported rates of 16 and $19 \%$ for propofol and ketamine groups, respectively. The intubation rate within $72 \mathrm{~h}$ of life reached $21 \%$ after propofol [25], 18-41\% in ketamine studies $[21,25,28]$, and $29 \%$ after fentanyl analgosedation [27]. Four studies without an unsedated arm described the second dose of surfactant requirements from 6.4 to $37.5 \%$, irrespectively of the choice of drug used (ketamine [25, 28], propofol [24, 25], or fentanyl [27]). The duration of MV and oxygen therapy was reported in $3[25,27,28]$ and 2 studies [27, 28], respectively, but lack of awake comparative precluded further interpretation. Pneumothoraxes occurred in $0 \%$ in Bourgoin et al. [21], $3 \%$ in Berneau et al. [28], and 4\% in Olivier et al. [27].
Comfort and Pain. Three studies described infant comfort or pain with different scales [21, 23, 25]. Dekker et al. [23] reported a significant reduction in the COMFORTneo score with analgosedation. Bourgoin et al. [21] and Brotelande et al. [25] evaluated pain with the Faceless Acute Neonatal Scale (FANS) score. Median scores were 1-2 [21, 25], when a score below 4 indicates comfort.

Clinical Tolerance. Krajewski et al. [22] reported for the analgosedation group a higher drop in oxygenation evaluated by changes in the $\mathrm{SpO}_{2} / \mathrm{FiO}_{2}$ ratio during LISA compared to baseline $(p<0.001)$. Procedural desaturation lasted significantly longer in the sedation group $(p<$ 0.001) in Dekker et al. [23]. Bradycardia did not occur more frequently in analgosedation groups $[22,23]$. Hypotension has been reported in 3 retrospective studies [23-25] using propofol. Dekker found no difference between sedated and unsedated patients [23]. When comparing propofol and ketamine, comparable drops of mean arterial blood pressure occurred but remained within physiological range [25]. Descamps et al. [24] reported hypotension in $14 \%$ of cases, which were transient and 
self-resolving. Incidence of apnoea and need for PPV during and immediately after procedure were significantly higher in sedated patients $(p=0.009$ and $p<0.001)[22$, 23 ]. Two cases of thoracic rigidity after fentanyl administration were reported [27]. Mortality rates before discharge ranged from 0 to $8 \%[21,25,28]$.

Technical Conditions. Bourgoin et al. [21] reported excellent or good quality in $83 \%$ of procedures after ketamine sedation, while in contrast, Krajewski et al. [22] noted no significant difference in the difficulty of the procedure between the sedated and unsedated groups. The duration of the procedure, as well as the number of attempts of catheter insertion was similar in both groups $[22,23,25]$.

Long-Term Outcomes. Evidence regarding intermediate- or long-term outcomes of analgosedation during LISA remains limited. Few data on the comorbidities of prematurity (such as bronchopulmonary dysplasia, retinopathy of prematurity, patent ductus arteriosus, necrotizing enterocolitis, and cystic periventricular leukomalacia) are available $[21,25,28]$. These are detailed in $\mathrm{Ta}$ ble 2. No study described the impact of the procedure on cerebral oxygenation or electrical brain activity.

\section{Discussion}

This systematic review, including 1 RCT and 7 cohort studies addressing analgosedation during LISA procedure, highlighted that analgesic or sedative drugs increased infant comfort with a limited impact on the clinical evolution. A higher occurrence of desaturation and need of PPV during procedure were reported in the only RCT [20] and in 2 cohort studies [22, 23]. Rates of intubation and need for MV were similar to the non-sedated arm when available. Caution is required when analysing this result. The largest prospective study described in this review reported a non-significant increase $(2.3 \%$ from $1 \%)$ of treatment failure with analgosedation even if exposed infants had a GA that was 2 weeks higher (31 4/7 vs. 29 5/7) [22].

Included studies can be compared to LISA studies without sedation reporting on populations with similar mean GA (29-31 weeks) [29-31]. These studies reported rates for $\mathrm{MV}$ at $72 \mathrm{~h}$ of life of $22-34 \%$, comparable to those from analgosedation studies. The higher rate of intubations in Bourgoin et al. [21] (41\% in which 7/19 infants were intubated before LISA for apnoea or insufficient sedation) could be explained by high cumulative doses of ketamine (mean $1.8 \pm 0.9 \mathrm{mg} / \mathrm{kg}$ ). The need for

A Systematic Review of Analgosedation before LISA additional doses of surfactant was not higher than in comparative studies (22-40\%) [29-31]. Other clinical outcomes such as comorbidities of prematurity do not seem influenced by analgosedation.

Up to now, the balance between long-term impact of the potential pain from brief laryngoscopy and the potential long-term impact of medication remain unknown. Prolonged use of opioids or benzodiazepine over a week is associated with an increased risk of abnormal neurodevelopmental outcome at 2 years [32]. Even if analgosedation improves infant comfort, the question of the most effective drug remains. The ideal drug would suppress pain and discomfort, while maintaining cardiorespiratory stability and having a minimal impact on the respiratory drive. Moreover, it would allow a rapid onset and offset and be safe in the long term $[6,14,33]$. To optimize the effect of the chosen drug, the time interval before procedure varies according to its pharmacokinetics properties.

Multiple drugs have been studied for analgesia or sedation during LISA. Surveys revealed that the most common choices of premedication were opioids (23-63\%), followed by propofol (5-23\%), benzodiazepines (5-23\%), ketamine (9\%), and, surprisingly, muscle relaxants, either as mono- or polytherapies [12, 16, 17, 34].

Fentanyl is the most commonly used opioid in NICU practice. It has analgesic, sedative, and anaesthetic properties that are 50-100 times more potent than morphine [35]. It has a rapid onset of action and minimal effect on haemodynamic [35]. Remifentanil is another synthetic opioid with a very rapid onset and offset of action [7, 9]. Both carry a risk of thoracic rigidity with rapid injection, which may limit their use $[7,9,27]$. Naloxone, their antagonist, could attenuate the respiratory drive depression generated by opioids. It had been used by Elmekkawi et al. to facilitate extubation in INSURE procedure with high efficacy and no adverse effects [36].

Propofol, used by Dekker et al. [23], Brotelande et al. [25], and Descamps et al. [24], is a purely sedative drug without specific analgesic effects. It is a common premedication for neonatal intubation or INSURE and still enable procedural pain control [37]. Propofol has a rapid onset with a short duration of action. It reduces airway reactivity and muscle tone in the upper respiratory tract [7]. It has been associated with bradycardia, desaturations, and prolonged hypotension in neonates $[9,38]$, whereas the included studies reported limited incidence of those side effects. A recent RCT comparing propofol and atracurium-sufentanil for nasotracheal intubation of children reporting no difference in 
risk of neurodevelopmental delay at 2-year follow-up [39].

Bourgoin et al. [21], Brotelande et al. [25], and Berneau et al. [28] studied ketamine, which has both sedative and analgesic properties $[5,40]$. It has rapid onset of action, short duration of action, and relatively safe respiratory and haemodynamic profiles [40].

Benzodiazepines, most commonly midazolam, were reported in the prospective study from Krajewski et al. [22] and are often been reported in surveys [12, 16, 34]. They have minor analgesic effect, along with potential respiratory depression and neurotoxicity and therefore could not be recommended $[38,41]$.

Non-pharmacological measures to reduce pain have also been reported $[5,16,17]$. Oral sucrose or glucose, known to provide analgesia during minor neonatal procedures [38, $42]$, was reported by $18-20 \%$ of physicians $[16,17]$.

Postural control and swaddling have been widely used during procedure. They have shown variable effectiveness in reducing pain and stress behavioural pain responses associated with painful procedures [38, 43]. It is clear that other effective non-pharmacological measures including rocking/holding, skin-to-skin, and breastfeeding could not be used during LISA procedure.

Technical conditions are often good or excellent with analgosedation. de Kort et al. [44] studied quality and response to LISA without sedation and found a low success rate of the first attempt (52\%) and frequently inadequate technical quality (41\%). However, these results may also reflect a lack of experience, as suggested by $72 \%$ of firstattempt successes for neonatologists [44].

This systematic literature research provides an overview of the premedication used for sedation for LISA with several methodological strengths. According to a predefined protocol registered in PROSPERO, we searched 4 databases with indexing terms as well as the grey literature. There were no limitations for inclusion in terms of language or study design to complement the findings of RCTs and provide evidence based on real-world data. Some limitations remain. Different designs and inhomogeneity of the studies precluded the realization of a meta-analysis. Secondly, most of the included studied did not have a comparative arm. While we compared their result with those of recent studies comparing LISA to INSURE, we could not control for unavoidable differences (such as population, technical conditions, type of surfactant used, etc.). Moreover, the lack of study specifically designed for respiratory outcomes and the possibility that existing studies were underpowered to assess these outcomes should be taken into account when interpreting these results.
The representativeness of the population might be an issue as extremely premature infants were under-represented despite the extensive use of LISA in this age group. Adverse effects of analgosedation for these infants may be different or even more important.

Finally, many studies are ongoing, as detailed in additional data. Seven are RCTs using fentanyl, ketamine, remifentanil, or propofol as analgosedative drugs. Study outcomes are very diverse. LISA limits the evaluation of facial expression and, regardless of pain, induces oxygen desaturation and sometimes bradycardia, therefore limiting the effectiveness of common pain scales. Milesi et al. [45] developed the FANS for use when the neonate's face is hidden by respiratory devices, but this scale also includes heart rate variations and oxygen desaturation. Other studies will compare sedation impact on stress, oxygenation changes, oxidative damage, and cerebral oxygenation.

\section{Conclusion}

LISA without analgosedation improves clinical outcomes but has the disadvantage of awake laryngoscopy. Concerns regarding analgosedation include respiratory depression, risk of failure, and potential loss of the benefits reported without it.

A significant pain reduction with analgosedation was described in the only RCT addressing the question. Except for higher risks of oxygen desaturation and more PPV during procedure, clinical outcomes were not different. Observational studies also reported reduction in pain at the expense of higher procedural rates of desaturation, apnoea, and ventilation.

Many questions remain about best drugs, optimal dosages, and long-term impact of these drugs, but those questions are not resolved for intubation either. Nine ongoing studies should provide welcome additional evidence to supplement the limited existing data.

\section{Statement of Ethics}

The research was conducted ethically in accordance with the Declaration of Helsinki ethical principles. The paper is exempt from Ethical Committee approval. All data were collected and synthesized from previous clinical trials for which informed consent had already been obtained by the trial investigators. The protocol was registered in advance of data extraction with the Prospective Register of Systematic Reviews (registered September 2020; CRD42020205365). 


\section{Conflict of Interest Statement}

The authors have no conflicts of interest to declare.

\section{Funding Sources}

This research received no specific grant from any funding agency.

\section{Author Contributions}

S.T., N.H., and D.S. contributed to the search strategy, data selection, and analysis. S.T. drafted and revised the manuscript. C.L. cooperated to the revision of the draft. V.R. helped with data interpretation and writing and editing of the manuscript. All the authors reviewed and approved the manuscript.

\section{Data Availability Statement}

All data generated or analysed are included in this article and its online supplementary material. Further enquiries can be directed to the corresponding author.

\section{References}

1 Bellos I, Fitrou G, Panza R, Pandita A. Comparative efficacy of methods for surfactant administration: a network meta-analysis. Arch Dis Child Fetal Neonatal Ed. 2021 Sep;106(5):474-87.

2 Rigo V, Lefebvre C, Broux I. Surfactant instillation in spontaneously breathing preterm infants: a systematic review and meta-analysis. Eur J Pediatr. 2016;175:1933-42.

3 Sweet D, Carnielli V, Greisen G, Hallman M, Ozek E, Te Pas A, et al. European consensus guidelines on the management of respiratory distress syndrome: 2019 update. Neonatology. 2019;115:432-50.

4 Herting E, Härtel C, Göpel W. Less invasive surfactant administration: best practices and unanswered questions. Curr Opin Pediatr. 2020;32:228-34.

5 Vento M, Bohlin K, Herting E, Roehr CC, Dargaville PA. Surfactant administration via thin catheter: a practical guide. Neonatology. 2019;116:211-26.

6 Balakrishnan A, Sanghera RS, Boyle EM. New techniques, new challenges: the dilemma of pain management for less invasive surfactant administration? Paediatric and Neo Pain. 2021;3(1):2-8.

7 Duncan HP, Zurick NJ, Wolf AR. Should we reconsider awake neonatal intubation? A review of the evidence and treatment strategies. Paediatr Anaesth. 2001;11:135-45.

8 Carbajal R, Eble B, Anand KJ. Premedication for tracheal intubation in neonates: confusion or controversy? Semin Perinatol. 2007;31:309-17.

9 Barrington K. Premedication for endotracheal intubation in the newborn infant. Paediatr Child Health. 2011;16:159.

10 Anand KJ; International Evidence-Based Group for Neonatal Pain. Consensus statement for the prevention and management of pain in the newborn. Arch Pediatr Adolesc Med. 2001;155:173-80.

11 Sawyer T, Johnson K. Neonatal intubation: past, present, and future. Neo Rev. 2020;21:e335-41.

12 Klotz D, Porcaro U, Fleck T, Fuchs H. European perspective on less invasive surfactant administration: a survey. Eur J Pediatr. 2017; 176:147-54
13 Kurepa D, Perveen S, Lipener Y, Kakkilaya V. The use of less invasive surfactant administration (LISA) in the United States with review of the literature. J Perinatol. 2019;39:426-32.

14 Peterson J, Den Boer MC, Roehr CC. To sedate or not to sedate for less invasive surfactant administration: an ethical approach. Neonatology. 2021;118(6):639.

15 Kasser S, Hartley C, Rickenbacher H, Klarer L, Depoorter A, Datta N, et al. Birth experience in newborn infants is associated with changes in nociceptive sensitivity. Sci Rep. 2019;9:4117.

16 Fernandez C, Boix H, Camba F, Comuñas JJ, Castillo F. Less invasive surfactant administration in Spain: a survey regarding its practice, the target population, and premedication use. Am J Perinatol. 2020;37:277-80.

17 Bhayat S, Kaur A, Premadeva I, Reynolds P, Gowda H. Survey of less invasive surfactant administration in England, slow adoption and variable practice. Acta Paediatr Int J Paediatr. 2020;109:505-10.

18 Göpel W, Kribs A, Ziegler A, Laux R, Hoehn $\mathrm{T}$, Wieg $\mathrm{C}$, et al. Avoidance of mechanical ventilation by surfactant treatment of spontaneously breathing preterm infants (AMV): an open-label, randomised, controlled trial. Lancet. 2011;378:1627-34

19 Page MJ, Mckenzie JE, Bossuyt PM, Boutron I, Hoffmann TC, Mulrow CD, et al. The PRISMA 2020 statement: an updated guideline for reporting systematic reviews. J Clin Epidemiol. 2021 Jun;134:178-89.

20 Dekker J, Lopriore E, van Zanten HA, Tan RNGB, Hooper SB, Te Pas AB. Sedation during minimal invasive surfactant therapy: a randomised controlled trial. Arch Dis Child Fetal Neonatal Ed. 2019;104:F378F383.

21 Bourgoin L, Caeymaex L, Decobert F, Jung C, Danan C, Durrmeyer X, et al. Administering atropine and ketamine before less invasive surfactant administration resulted in low pain scores in a prospective study of premature neonates. Acta Paediatr Int J Paediatr. 2018;107: 1184-90.
22 Krajewski P, Szpecht D, Hożejowski R. Premedication practices for less invasive surfactant administration: results from a nationwide cohort study. J Mater Fet Neo Med. 2020 Dec 25:1-5.

23 Dekker J, Lopriore E, Rijken M, Rijntjes-Jacobs E, Smits-Wintjens V, Te Pas A. Sedation during minimal invasive surfactant therapy in preterm infants. Neonatology. 2016;109:30813.

24 Descamps CS, Chevallier M, Ego A, Pin I, Epiard C, Debillon T. Propofol for sedation during less invasive surfactant administration in preterm infants. Arch Dis Child Fetal Neonatal Ed. 2017;102:F455.

25 Brotelande C, Milési C, Combes C, Durand S, Badr M, Cambonie G. Premedication with ketamine or propofol for less invasive surfactant administration (LISA): observational study in the delivery room. Eur J Pediatr. 2021 Sep;180(9):3053-8.

26 Chevallier M, Durrmeyer X, Ego A, Debillon T; PROLISA Study Group. Propofol versus placebo (with rescue with ketamine) before less invasive surfactant administration: study protocol for a multicenter, double-blind, placebo controlled trial (PROLISA). BMC Pediatr. 2020;20:199.

27 Olivier F, Nadeau S, Bélanger S, Julien AS, Massé E, Ali N, et al. Efficacy of minimally invasive surfactant therapy in moderate and late preterm infants: a multicentre randomized control trial. Paediatr Child Heal. 2017; 22:120-4

28 Berneau P, Thu TNP, Pladys P, Beuchée A. Impact of surfactant administration through a thin catheter in the delivery room: a quality control chart analysis coupled with a propensity score matched cohort study in preterm infants. PLoS One. 2018 Dec 12;1313(12): e0208252.

29 Seo MY, Shim GH, Chey MJ. Clinical outcomes of minimally invasive surfactant therapy via tracheal catheterization in neonates with a gestational age of 30 weeks or more diagnosed with respiratory distress syndrome. Neonatal Med. 2018;25(3):109-17. 
30 Dargaville P, Aiyappan A, De Paoli A, Kuschel CA, Kamlin CO, Carlin JB, et al. Minimally-invasive surfactant therapy in preterm infants on continuous positive airway pressure. Arch Dis Child Fetal Neonatal Ed. 2013 Mar;98(2):F122-6.

31 Aguar M, Cernada M, Brugada M, Gimeno A, Gutierrez A, Vento M. Minimally invasive surfactant therapy with a gastric tube is as effective as the intubation, surfactant, and extubation technique in preterm babies. Acta Paediatr Int J Paediatr. 2014 Jun;103(6):e229-33.

32 Puia-Dumitrescu M, Comstock BA, Li S, Heagerty PJ, Perez KM, Law JB, et al. Assessment of 2-year neurodevelopmental outcomes in extremely preterm infants receiving opioids and benzodiazepines. JAMA Netw Open. 2021;4:e2115998.

33 De Kort EH, Reiss IK, Simons SH. Sedation of newborn infants for the INSURE procedure, are we sure? Biomed Res Int. 2013;2013: 892974.

34 Heiring C, Jonsson B, Andersson S, Björklund LJ. Survey shows large differences between the Nordic countries in the use of less invasive surfactant administration. Acta Paediatr Int J Paediatr. 2017;106:382-6.
35 Thigpen JC, Odle BL, Harirforoosh S. Opioids: a review of pharmacokinetics and pharmacodynamics in neonates, infants, and children. Eur J Drug Metab Pharmacokinet. 2019; 44:591-609.

36 Elmekkawi A, Abdelgadir D, Van Dyk J Choudhury J, Dunn M. Use of naloxone to minimize extubation failure after premedication for INSURE procedure in preterm neonates. J Neonatal Perinatal Med. 2016;9:363-70.

37 Durrmeyer X, Breinig S, Claris O, Tourneux $\mathrm{P}$, Alexandre C, Saliba E, et al. Effect of atropine with propofol versus atropine with atracurium and sufentanil on oxygen desaturation in neonates requiring nonemergency intubation: a randomized clinical trial. JAMA. 2018;319:1790-801.

38 Committee on Fetus and Newborn and Section on Anesthesiology and Pain Medicine. Prevention and management of procedural pain in the neonate: an update. Pediatrics. 2016 Feb;137(2):e20154271.

39 Tauzin M, Marchand-Martin L, Lebeaux C, Breinig S, Claris O, Tourneux P, et al. Neurodevelopmental outcomes after premedication with atropine/propofol versus atropine/atracurium/sufentanil for neonatal intubation: 2 -year follow-up of a randomized clinical trial. J Pediatr. 2021;231:273-7.e3.
40 Bhutta AT. Ketamine: a controversial drug for neonates. Semin Perinatol. 2007;31(5):303-8.

$41 \mathrm{Ng}$ E, Taddio A, Ohlsson A. Intravenous midazolam infusion for sedation of infants in the neonatal intensive care unit. Cochrane Database Syst Rev. 2017 Jan 31;1(1):CD002052.

42 Stevens B, Yamada J, Ohlsson A, Haliburton S, Shorkey A. Sucrose for analgesia in newborn infants undergoing painful procedures. Cochrane Database Syst Rev. 2016 Jul 16;7(7): CD001069.

43 Hatfield LA, Murphy N, Karp K, Polomano RC. A systematic review of behavioral and environmental interventions for procedural pain management in preterm infants. J Pediatr Nurs. 2019;44:22-30.

44 de Kort E, Kusters S, Niemarkt H, van Pul C, Reiss I, Simons S, et al. Quality assessment and response to less invasive surfactant administration (LISA) without sedation. Pediatr Res. 2020;87:125-30.

45 Milesi C, Cambonie G, Jacquot A, Barbotte E, Mesnage R, Masson F, et al. Validation of a neonatal pain scale adapted to the new practices in caring for preterm newborns. Arch Dis Child Fetal Neonatal Ed. 2010;95:F263F266. 